\title{
Comparison of a blood-free medium and a filtration technique for the isolation of Campylobacter spp. from diarrhoeal stools of hospitalised patients in central Australia
}

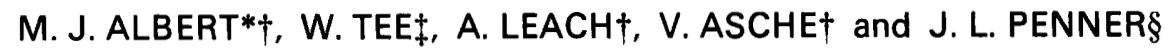 \\ - Alice Springs Hospital, Alice Springs, Northern Territory 0871, † Menzies School of Health Research, Casuarina, \\ Northern Territory 0811, $\ddagger$ Fairfield Infectious Diseases Hospital, Fairfield, Victoria 3078, Australia and \\ $\S$ University of Toronto, Toronto, Ontario M5S 1A8, Canada
}

\begin{abstract}
Summary. Single specimens of diarrhoeal stool from 676 patients, mostly aboriginals aged less than 5 years, admitted to Alice Springs Hospital, central Australia, for diarrhoea between Sept. 1988 and Feb. 1989, were examined for Campylobacter spp. by culture on a blood-free medium with selective supplement (BFM; Oxoid) and blood agar overlaid with a membrane filter (FM). Campylobacter spp. were isolated on either BFM or FM or both from 225 patients. Campylobacter spp. were isolated on BFM alone from 75 patients and on FM alone from 213 patients $\left(p<0.001 ; \chi^{2}\right.$ test). Most campylobacters isolated on BFM were $C$. jejuni. All $C$. jejuni subsp. doylei, all "C. upsaliensis" except one, all $C$. laridis, $C$. fetus subsp. fetus and several uncharacterised Campylobacter isolates were isolated on FM only. C. jejuni was isolated on BFM but not FM from several patients, and vice versa. Serotyping of $C$. jejuni and C. coli isolated from both media showed the serotypes recovered from the two media to be different in some patients. In some patients concurrent infection with several species or serotypes (up to five) of Campylobacter, or both, was shown for the first time by the use of FM. We conclude that the use in combination of a selective medium and a non-selective medium with a filtration technique are better than either medium alone for the isolation of Campylobacter spp.
\end{abstract}

\section{Introduction}

Both Campylobacter jejuni and C. coli have been shown to cause diarrhoea worldwide. ${ }^{1,2}$ Several solid media have been formulated for the isolation of these species from faeces, most of which contain a combination of antibiotics to suppress the normal faecal flora and are incubated at $42^{\circ} \mathrm{C}$ in a micro-aerophilic atmosphere. ${ }^{3-5}$ There are several reports that Campylobacter spp. other than $C$. jejuni and $C$. coli can cause diarrhoea, and these organisms have usually been isolated from diarrhoeal stools when an antibiotic-free medium that makes use of a filtration technique (filter medium, FM) has been used ${ }^{6-8}$ In this technique, a membrane filter of pore diameter 0.45 or $0.65 \mu \mathrm{m}$ is applied to the surface of a blood-agar plate and spot inoculated with a faecal suspension. Campylobacters are able to pass through the pores, whereas other organisms are held back. The filter paper is removed after $30 \mathrm{~min}$, and the plate is then incubated in a micro-aerophilic atmosphere. ${ }^{9}$

Received 7 Oct. 1991 ; accepted 17 Nov. 1991

* Present address to which correspondence should be sent: Dr M. J. Albert, ICDDR, B, GPO Box 128, Dhaka 1000, Bangladesh.
Another development in the isolation of Campylobacter spp., especially of $C$. jejuni and $C$. coli, is the formulation of a blood-free medium (BFM) in which blood is replaced by charcoal..$^{10.11}$ In a study of the aetiology and epidemiology of acute diarrhoea in central Australia, we compared the efficacies of BFM and FM for the isolation of Campylobacter spp.

\section{Materials and methods}

\section{Isolation of Campylobacter spp.}

Single samples of diarrhoeal stools from 676 patients, mostly aboriginals aged less than 5 years, were examined. These patients were admitted to hospital between Sep. 1988 and Feb. 1989. For culture of Campylobacter spp., BFM with cefoperazone supplement (Oxoid) and FM (sheep blood 5\% agar overlaid with a $0.65-\mu \mathrm{m}$ pore size Sartorius membrane filter) were used. The inoculated BFM was incubated at $42^{\circ} \mathrm{C}$ in an anaerobic jar with catalyst (BBL) in a micro-aerophilic atmosphere generated by a Campylobacter gas-generating sachet (Oxoid). The plate was examined after $48 \mathrm{~h}$ and, if no campylobacter was detected, was discarded. Initial evaluation suggested 
Table I. Comparison of isolation of Campylobacter spp. on BFM and FM from diarrhoeal stools of 676 patients in hospital in Alice Springs, central Australia for diarrhoea (Sep. 1988 to Feb. 1989)

\begin{tabular}{|c|c|c|c|c|c|c|c|c|c|c|c|c|c|c|c|c|c|}
\hline \multirow{2}{*}{$\begin{array}{l}\text { Category of } \\
\text { result }\end{array}$} & \multirow{2}{*}{$\begin{array}{l}\text { Total } \\
\text { number } \\
\text { of } \\
\text { patients }\end{array}$} & \multicolumn{8}{|c|}{$\begin{array}{l}\text { Number of patients from whom } \\
\text { Campylobacter spp. were isolated on BFM }\end{array}$} & \multicolumn{8}{|c|}{$\begin{array}{l}\text { Number of patients from whom } \\
\text { Campylobacter spp. were isolated on FM }\end{array}$} \\
\hline & & jej & col & doyl & ups & hyoint & lar & fetus & U.C. & jej & col & doyl & ups & hyoint & lar & fetus & U.C. \\
\hline $\begin{array}{l}\text { 1. Identical species } \\
\text { on both media }\end{array}$ & 19 & 14 & 0 & 0 & 0 & 2 & 0 & 0 & 3 & 14 & 0 & 0 & 0 & 2 & 0 & 0 & 3 \\
\hline $\begin{array}{l}\text { 2. One identical species } \\
\text { on both media and } \\
\text { additional species on } \\
\text { either medium }\end{array}$ & 21 & 18 & 2 & 0 & 1 & 0 & 0 & 0 & 1 & 18 & 4 & 10 & 10 & 0 & 0 & 0 & 3 \\
\hline $\begin{array}{l}\text { 3. One species on BFM } \\
\text { and one or more } \\
\text { species on FM }\end{array}$ & 23 & 19 & 1 & 0 & 0 & 2 & 0 & 0 & 1 & 3 & 0 & 15 & 6 & 0 & 0 & 0 & 6 \\
\hline $\begin{array}{l}\text { 4. C. jejuni on BFM } \\
\text { and no campylobacters } \\
\text { on FM }\end{array}$ & 12 & 12 & 0 & 0 & 0 & 0 & 0 & 0 & 0 & 0 & 0 & 0 & 0 & 0 & 0 & 0 & 0 \\
\hline $\begin{array}{l}\text { 5. No campylobacters on } \\
\text { BFM and one or more } \\
\text { Campylobacter spp. } \\
\text { on FM }\end{array}$ & 150 & 0 & 0 & 0 & 0 & 0 & 0 & 0 & 0 & 22 & 6 & 47 & 35 & 5 & 2 & 2 & 63 \\
\hline Total & 225 & 63 & 3 & 0 & 1 & 4 & 0 & 0 & 5 & 57 & 10 & 72 & 51 & 7 & 2 & 2 & 75 \\
\hline
\end{tabular}

BFM, blood-free medium with cefoperazone supplement (Oxoid); FM, blood agar overlaid with a membrane filter; jej, C. jejuni; col, $C$. coli; doyl, C. jejuni subsp. doylei; ups, "C. upsaliensis"; hyoint, C. hyointestinalis; lar, C. laridis; fetus, C. fetus subsp. fetus; U.C., uncharacterised Campylobacter sp.

that incubation of plates for longer than $48 \mathrm{~h}$ did not affect the isolation rates of Campylobacter spp. The inoculated FM was incubated at $37^{\circ} \mathrm{C}$ in a microaerophilic atmosphere generated as above. The plate was examined for campylobacters after $48 \mathrm{~h}$ and daily thereafter for 5 days. Different colony morphotypes were picked from both media for identification.

Campylobacter colonies were identified and speciated by the following tests: morphology after Gram's staining, oxidase and catalase production, nitrate reduction, hippurate hydrolysis, susceptibilities to nalidixic acid and cephalothin, micro-aerophilic growth at $42^{\circ} \mathrm{C}$ and $25^{\circ} \mathrm{C}$, aerobic growth at $37^{\circ} \mathrm{C}$, $\mathrm{H}_{2} \mathrm{~S}$ production in triple sugar iron agar (TSI), tolerance to glycine $1.0 \%$ and $\mathrm{NaCl} 3.5 \%$, and the rapid urease test as described previously. ${ }^{12}$ Speciation was further confirmed by electrophoresis of whole-cell proteins as described elsewhere. ${ }^{13} C$. jejuni NCTC 11351, C. jejuni subsp. doylei NCTC 11937, C. coli NCTC 11366, C. laridis NCTC 11352, C. fetus subsp. fetus NCTC 11842, C. fetus subsp. venerealis NCTC $11354, C$. hyointestinalis NCTC 11608, "C. upsaliensis" NCTC 11926 and NCTC 11541, and $C$. cryaerophila NCTC 11885 were used as reference strains.

\section{O-serotyping}

Antigenic extracts of $C$. jejuni and $C$. coli were prepared for O-serotyping according to the procedure described previously ${ }^{14}$ and sent to the Department of Microbiology, University of Toronto, Canada, where they were used to sensitise sheep erythrocytes for serotyping by the passive haemagglutination technique (PHA). The panel of antisera reported earlier ${ }^{15}$ was extended by including antisera against the recently defined O-serotypes O 60-0 66. The O-serotype assigned to an isolate was based on the antisera in which PHA titres greater than 40 were observed. Titres of 40 and 80 were considered weak $\left({ }_{w}\right)$ reactions.

\section{Examination for other enteric pathogens}

Parasites were sought by direct microscopy of a wet preparation of faeces and bacterial pathogens such as Salmonella, Shigella, Vibrio, Yersinia enterocolitica, Clostridium difficile, Aeromonas and Plesiomonas spp. were identified by standard methods as described elsewhere ${ }^{16}$ Rotavirus, astrovirus, and enteric adenoviruses 40 and 41 were identified by monoclonal antibody-based enzyme-linked immunosorbent assays $^{17-19}$ by $\mathrm{Dr}$ J. Herrmann, University of Massachusetts Medical Center, Worcester, MA, USA.

\section{Results}

Campylobacter spp. were isolated on BFM or FM or both media from the stools of $225(33.3 \%)$ of the 676 patients (table I). Campylobacter spp. were isolated on BFM alone from 75 patients and on FM alone from 213 patients, the difference being highly significant $\left(p<0.001 ; \chi^{2}\right.$ test). Most of the strains isolated on BFM were $C$. jejuni, a few were $C$. hyointestinalis, uncharacterised Campylobacter spp., and C. coli and one was " $C$. upsaliensis". On the other hand, FM yielded large numbers of uncharacterised Campylobacter spp. and " $C$. upsaliensis". C. jejuni subsp. doylei, C. laridis and $C$. fetus subsp. fetus were isolated from 72 , two and two patients respectively on FM only. Identical organisms were isolated on both media from only 19 patients (category 1). In 12 patients, C. jejuni were isolated on 
Table II. Patients with more than one species of Campylobacter isolated by FM from their diarrhoeal stool in Alice Springs, central Australia (Sep. 1988 to Feb. 1989)

\begin{tabular}{|c|c|}
\hline Combination of Campylobacter spp. & $\begin{array}{c}\text { Number of } \\
\text { patients }\end{array}$ \\
\hline 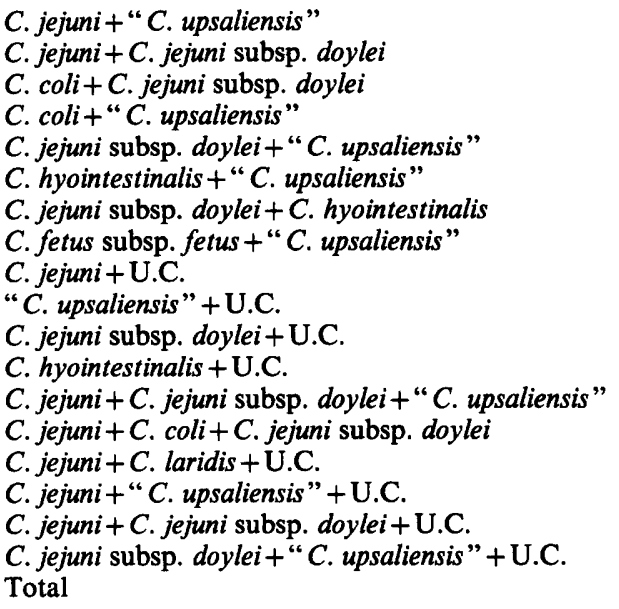 & $\begin{array}{r}6 \\
8 \\
1 \\
1 \\
7 \\
1 \\
1 \\
1 \\
3 \\
4 \\
10 \\
1 \\
4 \\
1 \\
1 \\
1 \\
1 \\
2 \\
54\end{array}$ \\
\hline
\end{tabular}

U.C., uncharacterised Campylobacter sp.

Table III. Comparison of $C$. jejuni and $C$. coli serotypes and concurrent isolates obtained from BFM and FM cultures from diarrhoeal stools of hospitalised patients in Alice Springs, central Australia (Sep. 1988 to Feb. 1989)

\begin{tabular}{|c|c|c|}
\hline $\begin{array}{l}\text { Patient } \\
\text { no. }\end{array}$ & $\begin{array}{l}\text { Organisms with } \\
\text { serotype on BFM }\end{array}$ & $\begin{array}{l}\text { Organisms with serotype } \\
\text { on FM }\end{array}$ \\
\hline 457 & C. jejuni $\mathrm{O} 22$ & C. jejuni $\mathrm{O} 22,64$ \\
\hline 488 & C. jejuni 08,17 & $\begin{array}{l}\text { C. jejuni } \mathrm{O} 8,17+C . \text { jejuni } \\
\text { subsp. doylei }\end{array}$ \\
\hline 565 & C. jejuni 08,17 & C. jejuni $08,17+$ C. jejuni 058 \\
\hline 579 & C. jejuni 08 & C. jejuni $\mathrm{O} 8,17+$ U.C. \\
\hline 589 & C. jejuni $\mathrm{Ol}, 44$ & C. jejuni $\mathrm{Ol}, 44$ \\
\hline 590 & C. jejuni $\mathrm{Ol}, 44$ & $\begin{array}{l}\text { C. jejuni } \mathrm{Ol}, 44+C . \text { jejuni } \\
\text { subsp. doylei }\end{array}$ \\
\hline 654 & C. coli $\mathrm{O} 54$ & $\begin{array}{l}\text { C. jejuni } \mathrm{O} 3_{\mathrm{w}}+C . \text { jejuni } \\
\text { O66+ “C. upsaliensis" }+C . \\
\text { jejuni subsp. doylei }\end{array}$ \\
\hline 648 & C. jejuni 08,17 & $\begin{array}{l}\text { C. jejuni } \mathrm{O} 8,17+C . \text { jejuni } \mathrm{O} 1 \text {, } \\
44+C . \text { jejuni } \mathrm{O} 22,64+" C . \\
\text { upsaliensis" }\end{array}$ \\
\hline 672 & C. jejuni $\mathrm{Ol}, 44$ & C. jejuni NT \\
\hline 695 & C. jejuni $\mathrm{Ol}, 44$ & C. jejuni 045 \\
\hline 724 & C. jejuni 019 & $\begin{array}{l}\text { C. jejuni } \mathrm{NT}+C . \text { jejuni subsp. } \\
\text { doylei }\end{array}$ \\
\hline 734 & C. coli $\mathrm{O} 54$ & $\begin{array}{l}\text { C. coli } \mathrm{O} 54+C . \text { jejuni subsp. } \\
\text { doylei }\end{array}$ \\
\hline 760 & C. jejuni NT & $\begin{array}{r}\text { C. jejuni } \mathrm{O} 41+C . \text { jejuni } \mathrm{O} 5 \\
31+C . \text { jejuni } \\
\text { subsp. doylei }\end{array}$ \\
\hline 970 & C. jejuni $\mathrm{O} 22$ & C. jejuni $\mathrm{O} 22$ \\
\hline
\end{tabular}

U.C., uncharacterised Campylobacter spp; ${ }_{w}$, weak reaction with the antiserum; NT, not typable.

BFM only (category 4). In category 3, fewer specimens yielded $C$. jejuni on FM than on BFM and in category 5 , this organism was isolated on FM in 22 patients, but on BFM in none. Uncharacterised Campylobacter spp. could not be completely identified because all except five isolates were lost after primary isolation; however, all of them had been confirmed as Campylobacter spp. because they had characteristic morph- ology after Gram's stain, were motile, and were catalase and oxidase positive.

Of the 225 patients from whom Campylobacter spp. were isolated, the faeces of 121 yielded no other recognised enteric pathogen. Of these 121 patients, "C. upsaliensis" was isolated as sole pathogen in 10 and together with another Campylobacter sp. in 15;C. jejuni subsp. doylei was isolated as sole pathogen in 12 and accompanied by another Campylobacter sp. in 23; and $C$. hyointestinalis was present as the only pathogen in two and accompanied by another Campylobacter sp. in four.

Of the 75 patients from whom a Campylobacter sp. was isolated on BFM, only one sample yielded more than one species on this medium (table I, category 2). On the other hand, the faeces of 54 of the 213 patients that were Campylobacter-positive on FM, yielded several species on this medium (table II).

Data on serotypes of $C$. jejuni or $C$. coli isolates from both media were available for 14 patients and these serotypes were compared with those of other Campylobacter spp. when present (table III). In five patients (nos. 488, 589, 590, 734 and 970) the same serotypes of C. jejuni or C. coli were isolated from both media, with or without additional Campylobacter spp. from FM. In two patients (nos. 565 and 648), $C$. jejuni serotypes other than those present on BFM were isolated. From the remaining seven patients, different serotypes were isolated on BFM and FM. Some patients in this series were infected with between three and five different Campylobacter spp. or serotypes.

\section{Discussion}

In this study, a significantly higher isolation rate, or more species of Campylobacter, or both, were obtained with a filtration technique than with a selective medium, as reported in previous studies., ${ }^{5,10}$ The incubation temperature for BFM recommended by the manufacturers is $42^{\circ} \mathrm{C}$, and we are not certain whether incubation of $\mathrm{FM}$ at $42^{\circ} \mathrm{C}$ instead of $37^{\circ} \mathrm{C}$ would have affected the isolation rate reported in this study. Faeces from 31 patients yielded $C$. jejuni on BFM, of which only three were also detected with FM (table I, categories 3 and 4). A similar but lower isolation rate of $C$. jejuni with FM has been reported previously? when the bacterial count was $<10^{5}$ organisms/g of faeces. It is likely that, in these patients, the organisms were present in smaller numbers. In the fifth category of patients (table I), faeces from 22 patients yielded $C$. jejuni on FM, none of which were positive with BFM. Either the selective supplement in the medium or the higher incubation temperature could have inhibited their growth on BFM. Such atypical $C$. jejuni strains have been encountered in other studies. ${ }^{6}, 12$

The Campylobacter spp. recovered on FM only were all $C$. jejuni subsp. doylei, except for one " $C$. upsaliensis" isolate, one C. laridis and one C. fetus subsp. fetus. Some $C$. hyointestinalis strains were 
isolated from FM only. Of these species, only $C$. laridis is a known diarrhoeal pathogen $;{ }^{20}$ the other species are suspected diarrhoeal pathogens. ${ }^{7,8,20-23}$ However, increasing importance has been attached to $C$. jejuni subsp. doylei and " $C$. upsaliensis" as possible diarrhoeal pathogens. Although the former was present in 72 patients, it was isolated as sole pathogen in 12 . The latter was isolated as the only pathogen in 10 of the 51 patients in whose faeces this organism was found.

Case-control studies are needed to establish unequivocally the role of these various species as diarrhoeal pathogens. The 75 unidentified campylobacters isolated from FM were the largest group of organisms to be isolated with this medium. The fact that all except five were lost after primary isolation suggests that they were extremely fragile organisms and great care should be taken in future to define optimal conditions for their isolation and maintenance. The five viable strains could not be assigned to any recognised species of Campylobacter and further studies are in progress. We may have encountered new species of Campylobacter in this population.

We have demonstrated that several patients were infected with up to five different species or serotypes or both of Campylobacter. Moreover, the serotyping data

\section{References}

1. Blaser MJ, Reller LB. Campylobacter enteritis. $N$ Engl $J$ Med $1981 ; 305$ : 1444-1452.

2. Blaser MJ, Taylor DN, Feldman RA. Epidemiology of Campylobacter jejuni infections. Epidemiol Rev 1983; 5: 157-176.

3. Blaser MJ, Berkowitz ID, LaForce FM, Cravens J, Reller LB, Wang W-LL. Campylobacter enteritis : clinical and epidemiologic features. Ann Intern Med 1979; 91 : 179-185.

4. Bolton FJ, Robertson L. A selective medium for isolating Campylobacter jejuni/coli. J Clin Pathol 1982; 35: 462-467.

5. Goossens H, DeBoeck M, Butzler JP. A new selective medium for the isolation of Campylobacter jejuni from human faeces. Eur J Clin Microbiol 1983; 2: 389-394.

6. Bolton FJ, Hutchinson DN, Parker G. Reassessment of selective agars and filtration techniques for isolation of Campylobacter species from faeces. Eur J Clin Microbiol Infect Dis $1988 ; 7: 155-160$.

7. Goossens H, Vlaes L, DeBoeck $M$ et al. Is "Campylobacter upsaliensis" an unrecognised cause of human diarrhoea? Lancet 1990; 335: 584-586.

8. Steele TW, Sangster N, Lanser JA. DNA relatedness and biochemical features of Campylobacter spp. isolated in Central and South Australia. J Clin Microbiol 1985; 22: 71-74.

9. Steele TW, McDermott SN. The use of membrane filters applied directly to the surface of agar plates for the isolation of Campylobacter jejuni from faeces. Pathology 1984; 16: 263-265.

10. Bolton FJ, Hutchinson DN, Coates D. Blood-free selective medium for isolation of Campylobacter jejuni from feces. J Clin Microbiol 1984; 19: 169-171.

11. Karmali MA, Simor AE, Roscoe M, Fleming PC, Smith SS, Lane J. Evaluation of a blood-free, charcoal-based, selective medium for the isolation of Campylobacter organisms from feces. J Clin Microbiol 1986; 23: 456-459.

12. Tee W, Anderson BN, Ross BC, Dwyer B. Atypical campylobacters associated with gastroenteritis. J Clin Microbiol 1987; 25 : 1248-1252. on $C$. jejuni suggested for the first time that, sometimes, the serotypes of this species recovered from both media of the same patients were different. All these data, together with the high isolation rate of Campylobacter spp. (33.3\%) suggest that the ecology of Campylobacter spp. in this population is unusual and that the level of exposure of this population to this group of organisms is very high. Furthermore, the present study, as the previous studies, ${ }^{6,7}$ suggested that the use of a single selective medium is not satisfactory for the optimum isolation of Campylobacter spp. Although the FM alone yielded a higher isolation rate of Campylobacter spp., this medium also failed to isolate several strains of $C$. jejuni, which suggests that, for optimum isolation of Campylobacter spp. from diarrhoeal stools, a combination of media, particularly a selective medium and a non-selective medium employing a filtration technique, are needed.

We thank Diana Tomkins, Menzies School of Health Research (MSHR) for help with the research, J. Mathews, MSHR, and P. Bradford and M. Tyrrell, Department of Health, Alice Springs, for support, and Priyatosh Sukul, ICDDR, B, for typing the manuscript. J. L. Penner acknowledges the support of the Natural Sciences and Engineering Research Council of Canada.

13. Costas M, Owen RJ, Jackman PJH. Classification of Campylobacter sputorum and allied campylobacters based on numerical analysis of electrophoretic protein patterns. Syst Appl Microbiol 1987; 9: 125-131.

14. Lastovica AJ, Marshall RB, Penner JL. Campylobacter serotyping on shipped antigen extracts. Lancet 1986; 1: 320.

15. Penner JL, Hennessy JN, Congi RV. Serotyping of Campylobacter jejuni and Campylobacter coli on the basis of thermostable antigens. Eur J Clin Microbiol 1983; 2: 378-383.

16. World Health Organization. Programme for control of diarrhoeal diseases [CDD/83.3]. In: Manual for laboratory investigations of acute enteric infections. Geneva, World Health Organization. 1983.

17. Herrmann JE, Perron DM, Blacklow NR et al. Enzyme immunoassay with monoclonal antibodies for the detection of rotavirus in stool specimens. $J$ Infect Dis 1985; 152: 830-832.

18. Herrmann JE, Perron-Henry DM, Blacklow NR. Antigen detection with monoclonal antibodies for the diagnosis of adenovirus gastroenteritis. $J$ Infect Dis 1987; 155: $1167-1171$

19. Herrmann JE, Nowak NA, Perron-Henry DM, Hudson RW, Cubitt WD, Blacklow NR. Diagnosis of astrovirus gastroenteritis by antigen detection with monoclonal antibodies. J Infect Dis 1990; 161: 226-229.

20. Simor AE, Wilcox L. Enteritis associated with Campylobacter laridis. J Clin Microbiol 1987; 25: 10-12.

21. Edmonds P, Patton CM, Griffin PM et al. Campylobacter hyointestinalis associated with human gastrointestinal disease in the United States. J Clin Microbiol 1987; 25: 685-691.

22. Harvey SM, Greenwood JR. Probable Campylobacter fetus subsp. fetus gastroenteritis. J Clin Microbiol 1983; 18: 1278-1279.

23. Steele TW, Owen RJ. Campylobacter jejuni subsp. doylei subsp. nov., a subspecies of nitrate-negative campylobacters isolated from human clinical specimens. Int J Syst Bacteriol 1988; 38: 316-318. 